\title{
Dieta do cascudo Aspidoras fuscoguttatus (Ostariophysi, Callichthyidae) em riachos com diferentes características limnológicas e estruturais
}

\author{
Lilian Casatti, ${ }^{1,2}$,José Luis Veronezi Júnior ${ }^{1}$ \& Cristiane de Paula Ferreira ${ }^{1}$ \\ ${ }^{1}$ Laboratório de Ictiologia, Departamento de Zoologia e Botânica, IBILCE, \\ Universidade Estadual Paulista - UNESP, \\ Rua Cristóvão Colombo, 2265, Jardim Nazareth, CEP 15054-000, São José do Rio Preto, SP, Brasil, \\ e-mail:j_veronezi@yahoo.com.br,cristianebio@gmail.com \\ ${ }^{2}$ Autor para correspondência: Lilian Casatti,e-mail: lcasatti@ibilce.unesp.br
}

CASATTI, L., VERONEZI JÚNIOR, J.L. \& FERREIRA, C.P. Diet of the armored catfish Aspidoras fuscoguttatus (Ostariophysi, Callichthyidae) in streams with different limnological and structural features. Biota Neotrop. 9(1): http://www.biotaneotropica.org.br/v9n1/en/abstract?article+bn02109012009.

\begin{abstract}
In the present study Aspidoras fuscoguttatus Nijssen \& Isbrücker, 1976 diet was investigated based on specimens from 18 streams of São Paulo State northwestern region, upper Rio Paraná system. Stomach contents of 246 specimens were analyzed, with the registration of 26 types of feeding items showing high predominance of the autochthonous ones. Of these, Chironomidae, Simuliidae, and Ceratopogonidae aquatic larvae were the most important items, but the contribution of detritus, testate amoebas, and vegetal debris changed according to streams features. Whereas in clear waters feeding items can be visually selected by fishes, in turbid waters this selection may not occur. Thus, the species' ability in taking resources in sandy streams with turbid waters and the abundance of resources (Chironomidae larvae) are factors that seem to explain its high abundance and broad occurrence in the region.
\end{abstract}

Keywords: diet, Chironomidae, organic matter, vegetal debris, tecamoeba.

CASATTI, L., VERONEZI JÚNIOR, J.L. \& FERREIRA, C.P. Dieta do cascudo Aspidoras fuscoguttatus (Ostariophysi, Callichthyidae) em riachos com diferentes características limnológicas e estruturais. Biota Neotrop. 9(1): http://www.biotaneotropica.org.br/v9n1/pt/abstract?article+bn02109012009.

Resumo: No presente estudo a dieta de Aspidoras fuscoguttatus Nijssen \& Isbrücker, 1976 foi analisada em exemplares procedentes de 18 riachos da região noroeste do estado de São Paulo, sistema do Alto Rio Paraná. Foram analisados os conteúdos gástricos de 246 indivíduos, com o registro de 26 tipos de itens alimentares e predominância de itens autóctones. Destes, larvas de Chironomidae, Simuliidae e Ceratopogonidae foram os mais importantes, mas a contribuição de detritos, tecamebas e fragmentos de vegetais variou de acordo com as características dos riachos. Enquanto em águas mais claras os itens alimentares podem ser visualmente selecionados pelos peixes, em águas mais turvas essa seleção pode ocorrer. Assim, a habilidade da espécie em obter recursos em riachos com fundos arenosos e águas turvas e a abundância de recursos (larvas de Chironomidae) são fatores que parecem explicar a elevada abundância e ampla ocorrência da espécie na região.

Palavras-chave: dieta, Chironomidae, matéria orgânica, fragmentos de vegetais, tecamebas. 


\section{Introdução}

A substituição da vegetação nativa por pastagens e outras atividades agroeconômicas - que invariavelmente se estendem às áreas ripárias - afetam diretamente a biota aquática, sendo que os riachos são os primeiros ambientes a serem afetados por este tipo de impacto, em razão de seus reduzidos volumes de água e de sua grande interface com o meio terrestre (Menezes et al. 1990). A substituição da floresta ripária por pastagens pode diminuir a oferta de matéria orgânica alóctone, ocasionar mudanças nos hábitos alimentares dos peixes (Claro Jr. et al. 2004) e até mesmo indicar alterações nas proporções em biomassa de peixes insetívoros e herbívoros-detritívoros (Esteves et al. 2008).

Diante desse cenário de ininterruptas e amplas alterações em pequenos cursos d'água e áreas adjacentes, torna-se necessário conhecer e definir exigências e tolerâncias das principais espécies de peixes desses ambientes, principalmente para subsidiar estratégias adequadas à conservação e ao manejo da ictiofauna, inclusive visando futuros projetos de restauração (Rosenfeld 2003). Tal conhecimento é ainda mais relevante em áreas seriamente impactadas por ações antrópicas, como é o caso da região noroeste do estado de São Paulo. Nesta área restam 5\% da vegetação nativa (SMA/IF 2005), as pastagens representam de 70 a $75 \%$ do uso do solo (Silva et al. 2007) e estão sendo rapidamente convertidas em monoculturas de cana-de-açúcar, no sentido de suprir novas unidades produtoras de açúcar e álcool (Novaes 2007), representando uma das áreas mais críticas em termos de grau de preservação da ictiofauna (Casatti et al. 2008).

Como produto de recente inventário da ictiofauna realizado por nossa equipe em 95 riachos dessa região, Aspidoras fuscoguttatus Nijssen \& Isbrücker 1976, um cascudo que ocorre em trechos superiores e médios de riachos no sistema do Alto Rio Paraná (Garutti 1988, Reis et al. 2003), foi identificada como a quarta espécie mais abundante, perfazendo $7,5 \%$ do total de indivíduos coletados e ocorrendo em $76 \%$ dos riachos estudados. Apesar da sistemática de Aspidoras ser tema central de publicações recentes (Britto 1998, 2000, Britto et al. 2002, 2005), o conhecimento sobre a biologia de suas espécies ainda é incipiente, com a maioria das informações disponíveis registrada em condições artificiais (Burgess 1989). Exceções são os estudos naturalísticos sobre aspectos da história de vida de A. fuscoguttatus, realizados por Araujo \& Garutti (2002, 2003). Assim, em vista da elevada abundância e ampla ocorrência dessa espécie em riachos da região noroeste do estado de São Paulo, investigamos se sua alimentação varia conforme características estruturais e limnológicas dos riachos.

\section{1. Área de estudo}

A área de estudo (Figura 1) está situada na região noroeste do Planalto Ocidental Paulista e inclui as Unidades de Gerenciamento de Recursos Hídricos (UGRHI) São José dos Dourados e Turvo-Grande. O relevo da região é considerado suave, ondulado e razoavelmente uniforme (Arid 1973). O clima é tropical quente (Nimer 1989), com duas estações pluviométricas bem definidas: chuvosa, de outubro a março, e seca, de abril a setembro. Na primeira, a concentração de chuvas de verão é acentuada, acima de $80 \%$ da pluviometria total anual (Barcha \& Arid 1971).

\section{Material e Métodos}

Foram selecionados 18 riachos (Figura 1) com características físico-químicas e estruturais distintas, evidenciadas por 12 descritores (Apêndice 1). A composição do substrato foi determinada visualmente em campo, estimando-se a porcentagem de argila, areia, cascalho, rochas e laje ao longo do leito do trecho amostrado; a porcentagem de troncos/galhos e vegetação aquática (Poaceae) no hábitat interno

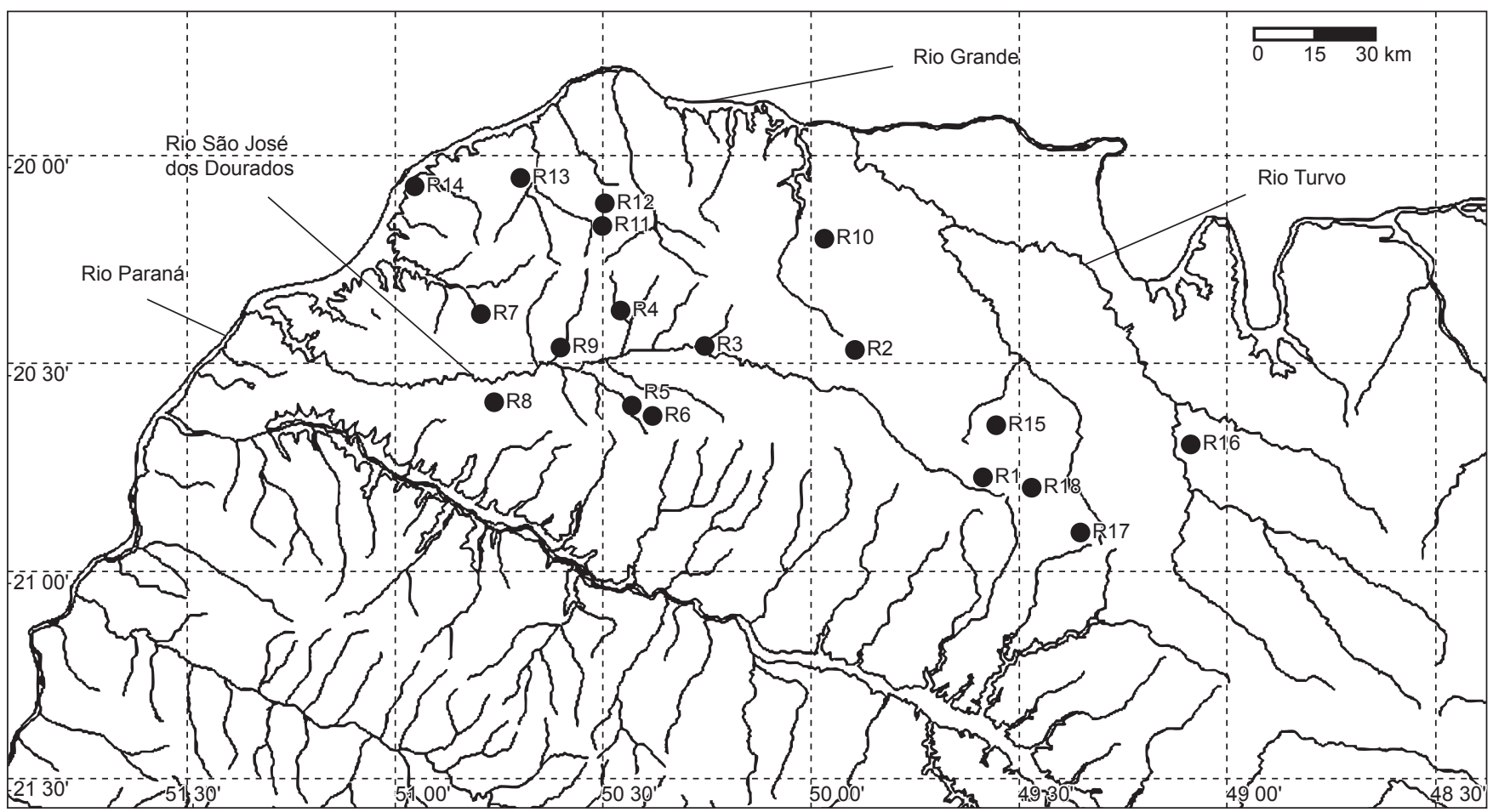

Figura 1. Localização geográfica dos riachos estudados (R1-R18) na região noroeste do estado de São Paulo, sistema do Alto Rio Paraná.

Figure 1. Geographical location of the studied streams (R1-R18) in the northwester region of the São Paulo State, upper Rio Paraná system. 
foi estimada visualmente de acordo com a ocupação da área amostral por estes elementos; as porcentagens de arbustos e de árvores no hábitat ripário foram estimadas de acordo com a presença destes elementos na zona ripária (30 m de largura) das duas margens do trecho amostral; a correnteza foi obtida por meio de um fluxômetro mecânico com três réplicas por meso-hábitat e posteriormente categorizada $(1=0-20 \mathrm{~cm} / \mathrm{s}, 2=20-40 \mathrm{~cm} / \mathrm{s}$, ou $3=40-60 \mathrm{~cm} / \mathrm{s}) ;$ a área amostral foi calculada com base nas medidas obtidas em vários transectos de largura e na extensão do trecho amostral; a turbidez foi medida diretamente em campo, empregando-se equipamento

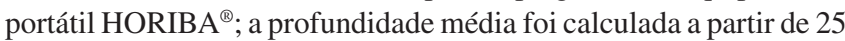
a 30 medidas tomadas ao longo de cada trecho amostral.

Os dados ambientais, bem como os peixes, foram coletados em uma ocasião, durante o dia, nos períodos secos de 2003, 2004 e 2005. $\mathrm{Na}$ coleta dos peixes, os limites superior e inferior de cada trecho amostral, contendo 75 metros de extensão, foram bloqueados com redes de malha $5 \mathrm{~mm}$ e, a seguir, foram aplicadas duas passagens de pesca elétrica $(220 \mathrm{~V}$ de corrente alternada, com 50-60 Hz, 3,4-4,1 A e $1000 \mathrm{~W}$, somando de 40-50 minutos de amostragem em cada trecho), rio acima, com intervalos de 20 minutos entre a primeira e a segunda passagem (Casatti et al. 2006). Após a coleta, os peixes foram fixados em formalina a $10 \%$ por aproximadamente 72 horas e posteriormente transferidos para solução de etanol a 70\%. Todos os exemplares estão depositados na coleção de Peixes do Departamento de Zoologia (DZSJRP) do Instituto de Biociências Letras e Ciências Exatas, IBILCE - UNESP, São José do Rio Preto, SP, Brasil.

Para a análise de dieta, foram selecionados de nove a 15 exemplares adultos de A. fuscoguttatus de cada riacho. Após a remoção dos estômagos, os itens alimentares foram triados e identificados, buscando a resolução taxonômica mais detalhada possível, com auxílio de literatura especializada e consulta a especialistas. Para cada item alimentar foram calculadas a composição percentual por números, a freqüência de ocorrência (Gelwick \& Matthews 1996) e a dominância (Hynes 1950). A dominância (eixo y) foi plotada em um gráfico em função da freqüência de ocorrência (eixo x), segundo a proposta de Bennemann et al. (2006), e os itens principais na dieta da espécie em cada riacho foram aqueles localizados no quadrante superior direito.

A dieta de $A$. fuscoguttatus nos riachos estudados foi exploratoriamente avaliada por meio de uma técnica de ordenação, a análise de escalonamento multidimensional não-métrica (NMDS) (Clarke \& Warwick 2001). Para tal, os valores de composição percentual dos itens agrupados (algas, microcrustáceos, insetos aquáticos, tecamebas, fragmentos de vegetais superiores, aracnídeos, insetos terrestres, detrito) foram utilizados para cálculo da matriz de similaridade por meio do coeficiente de Bray-Curtis, utilizando-se o programa estatístico PRIMER 6 (Clarke \& Gorley 2006). Um dos produtos da NMDS é distorção (ou stress) entre a matriz de similaridade e a ordenação produzida na representação gráfica dos eixos (Clarke \& Warwick 2001).

A hipótese nula de que os descritores do hábitat não exercem influências significativas na dieta de A. fuscoguttatus foi testada por meio de uma análise de similaridade (ANOSIM), que consiste em uma técnica não paramétrica, análoga à análise de variância (Clarke \& Warwick 2001), e foi processada através do programa estatístico PRIMER 6. Nesta análise, calculou-se o valor de R, uma medida comparativa do grau de separação das amostras, que amparou a decisão de aceite ou rejeição da hipótese nula. A amplitude de variação de $\mathrm{R}$ é de -1 a 1 , sendo que valores próximos a zero significam aceite da hipótese nula, e a probabilidade (p) representa a porcentagem de risco de ocorrer erro Tipo I segundo a nomenclatura estatística (Clarke \& Warwick 2001).

\section{Resultados}

Dentre os 246 exemplares examinados, 42 apresentaram estômagos vazios. Foram identificados 26 tipos de itens alimentares, a maioria de origem autóctone (Tabela 1). Reunindo todos os riachos em uma única análise, a predominância numérica dos itens autóctones se repete ( $81,4 \%$ contra $12,4 \%$ de origem alóctone e $6,2 \%$ de origem desconhecida, Tabela 1), dos quais larvas de Chironomidae e fragmentos de larvas de insetos se destacam em freqüência e dominância (Tabela 1). Com a análise conduzida separadamente por riacho, insetos aquáticos representaram o principal item em sete riachos (R3, R5, R7, R12, R14, R15, R17, Figuras 2 e 3), sendo que larvas de Chironomidae foram registradas nas amostras de todos os riachos (Figuras 2 e 3 ).

A ordenação dos riachos com base na composição percentual da dieta mostra três grupos de riachos (Figura 4a), discriminados de acordo com a participação de tecamebas, fragmentos de vegetais e detritos (Figuras 4b-d), visto que a participação de insetos aquáticos

Tabela 1. Composição percentual por número $(\mathrm{CP})$, frequiência de ocorrência (FO) e dominância (d) dos itens registrados nos conteúdos estomacais de Aspidoras fuscoguttatus procedentes de 18 riachos da região noroeste do estado de São Paulo, sistema do Alto Rio Paraná.

Table 1. Percent composition by number (CP), frequency of occurrence (FO), and dominance (D) of the feeding items registered in the stomach contents of Aspidoras fuscoguttatus from 18 streams in the São Paulo State northwestern region, upper Rio Paraná system.

\begin{tabular}{|c|c|c|c|}
\hline Itens & $\mathbf{C P}$ & $\begin{array}{l}\text { FO } \\
(\%)\end{array}$ & D \\
\hline \multicolumn{4}{|l|}{ Autóctones } \\
\hline 1. Cyanophyceae & 0,6 & 2,0 & 0 \\
\hline 2. Spyrogira & 0,6 & 2,0 & 0 \\
\hline 3. Larvas de Diptera não identificadas & 0,5 & 1,5 & 0 \\
\hline 4. Pupas de Diptera & 2,0 & 19,7 & 0 \\
\hline 5. Larvas de Chironomidae & 23,3 & 73,9 & 39,9 \\
\hline 6. Larvas de Ceratopogonidae & 5,9 & 18,7 & 0,5 \\
\hline 7. Larvas de Simuliidae & 5,0 & 15,8 & 3,4 \\
\hline 8. Pupas de Simuliidae & 2,0 & 6,4 & 0,5 \\
\hline 9. Larvas de Coleoptera & 1,4 & 4,4 & 2,0 \\
\hline 10. Larvas de Trichoptera & 4,8 & 15,3 & 2,0 \\
\hline 11. Fragmentos de larvas & 19,4 & 61,6 & 30,0 \\
\hline 12. Ninfas de Odonata & 1,6 & 4,9 & 0 \\
\hline 13. Ninfas de Ephemeroptera & 6,2 & 19,7 & 3,9 \\
\hline 14. Tecamebas & 7,9 & 25,1 & 1,5 \\
\hline 15. Crustáceos & 0,3 & 1,0 & 0 \\
\hline \multicolumn{4}{|l|}{ Alóctones } \\
\hline 16. Fragmentos vegetais & 4,8 & 15,3 & 0,5 \\
\hline 17. Aranae & 0,3 & 1,0 & 0 \\
\hline 18. Isoptera & 0,2 & 0,5 & 0 \\
\hline 19. Diptera & 0,5 & 1,5 & 0 \\
\hline 20. Orthoptera & 0,2 & 0,5 & 0 \\
\hline 21. Coleoptera & 0,8 & 2,5 & 0 \\
\hline 22. Formicidae & 0,2 & 0,5 & 0 \\
\hline 23. Hymenoptera & 0,2 & 0,5 & 0 \\
\hline 24. Fragmentos de insetos & 5,4 & 17,2 & 5,9 \\
\hline Origem indeterminada & & 0,0 & 0 \\
\hline 25. Fragmentos não identificados & 0,5 & 1,5 & 0,5 \\
\hline 26. Detritos & 5,7 & 18,2 & 9,4 \\
\hline
\end{tabular}


Casatti, L. et al.
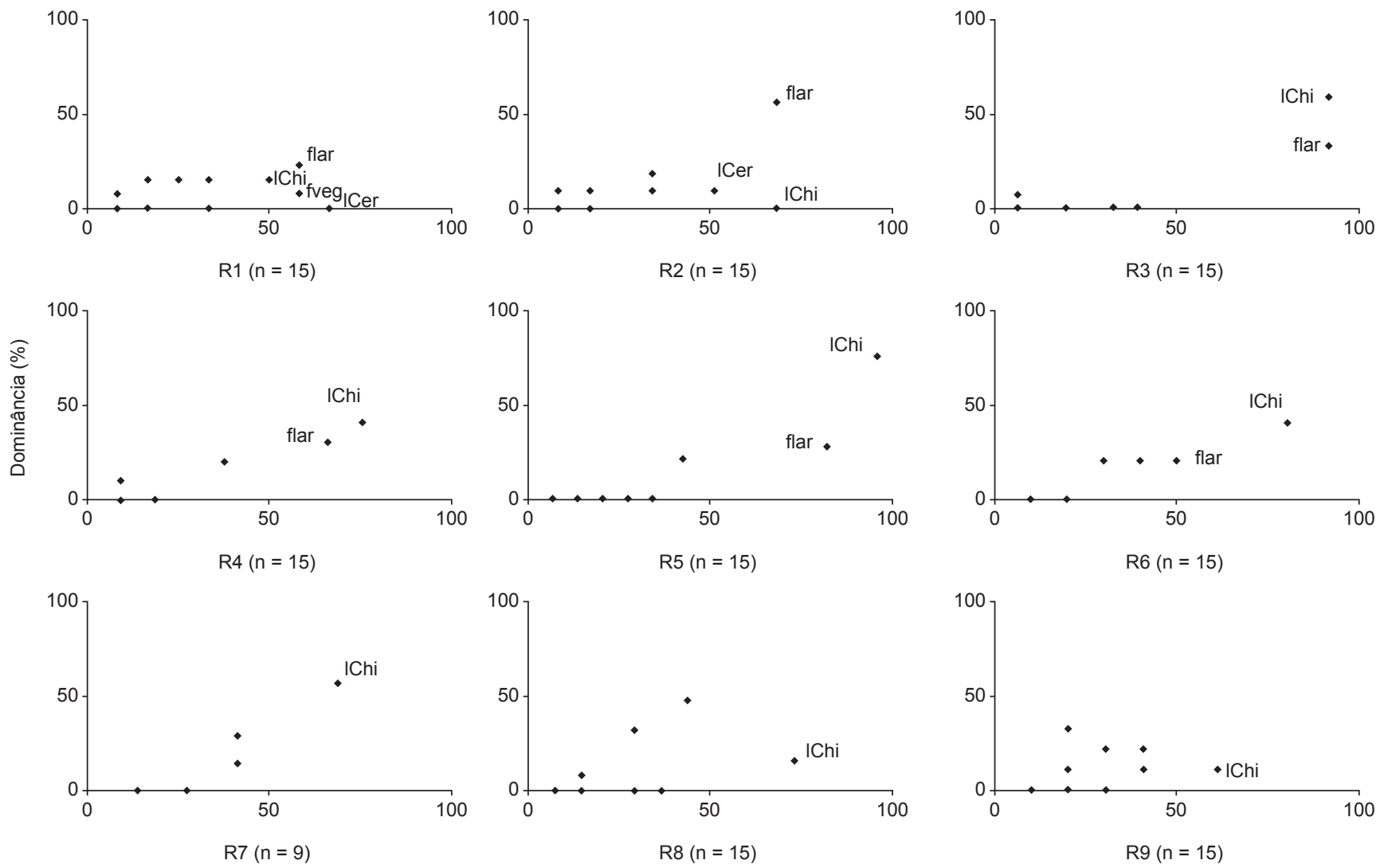

Frequência de ocorrência (\%)

Figura 2. Representação gráfica da dominância (eixo y) em função da freqüência de ocorrência (eixo x) dos itens alimentares encontrados nos conteúdos gástricos de Aspidoras fuscogutattus nos riachos R1 a R9 ( $\mathrm{n}=$ número de exemplares examinados): flar, fragmentos de larvas aquáticas de insetos; fveg, fragmentos de vegetais; 1Chi, larvas aquáticas de Chironomidae; 1Cer, larvas aquáticas de Ceratopogonidae.

Figure 2. Graphic representation of the dominance (axis y) in function to the frequency of occurrence (axis x) of the feeding items registered in the stomach contents of Aspidoras fuscogutattus in the streams R1 to R9 ( $\mathrm{n}=$ number of examined specimens): flar, aquatic insect larvae debris; 1Chi, aquatic Chironomidae larvae; 1Sim, aquatic Simuliidae larvae; 1Tri, aquatic Trichoptera larvae; nEph, Ephemeroptera nymphs; tec, testate amoebas; det, detritus.

na dieta da espécie foi elevada em praticamente todos os riachos (Figura 4e). O stress calculado foi de 0,15 , o que garante boa confiabilidade na interpretação dos resultados. Como resultado da ANOSIM (Tabela 2), obteve-se que a turbidez foi o descritor que demonstrou maior influência na dieta de A. fuscoguttatus. Considerando os itens com maior influência na discriminação dos grupos de riachos, as maiores proporções de tecamebas (Figura 4b) ocorreram em peixes procedentes de riachos com águas pouco turvas (R3, R10, R15-R17, mediana turbidez $=4 \mathrm{NTU}$ ) e com alguma vegetação ripária; as maiores proporções de fragmentos de vegetais (Figura 4c) foram registradas em peixes procedentes de riachos com águas relativamente mais turvas (R1, R5, R6, R14, mediana turbidez = 112 NTU) e com bancos de gramíneas junto das margens ou com cascalhos e rochas no fundo; e as maiores proporções de detritos (Figura 4d) em peixes de riachos com águas moderadamente turvas (R2, R4, R7-R9, R11-R13, $\mathrm{R} 18$, mediana turbidez $=16 \mathrm{NTU}$ ) e de fundo arenoso.

\section{Discussão}

Os riachos estudados são, de modo geral, rasos, pouco correntosos e pouco diversificados na composição do substrato, sendo que a baixa representatividade de árvores no hábitat ripário se reflete na baixa disponibilidade de troncos e galhos submersos. Nestes ambientes, a dieta de $A$. fuscoguttatus foi predominantemente composta por itens autóctones, representados por larvas aquáticas de insetos, notadamente Chironomidae, um dos grupos de insetos mais comumente registrados em ambientes lóticos (Carvalho \& Uieda 2004, 2006, Hay et al. 2008). Outras espécies da família Callichthyidae também apresentam dieta semelhante, com elevada participação de larvas aquáticas de insetos, como descrito para Corydoras gr. carlae Nijssen \& Isbrücker 1983 (Aranha et al. 1993), C. barbatus (Quoy \& Gaimard 1824) (Aranha et al. 1998) e C. aeneus (Gill 1858) (Aranha et al. 1993, Casatti 2002), por exemplo.

A maioria dos riachos estudados estava repleta de areia junto ao leito e tal condição pode ser desfavorável às atividades alimentares para um grande número de espécies de Siluriformes, (Lowe-McConnell 1987). Os callictídeos, contudo, utilizam barbilhões táteis para localizarem suas presas em meio ao substrato, que é revolvido superficialmente (Hahn et al. 1997, Aranha et al. 1998, Casatti 2002) e, por isso, são conhecidos como especuladores de substrato ("hunting by speculation", Curio 1976, ou "grubber excavating while moving”, Sazima 1986). Portanto, tais características 
Alimentação de Aspidoras
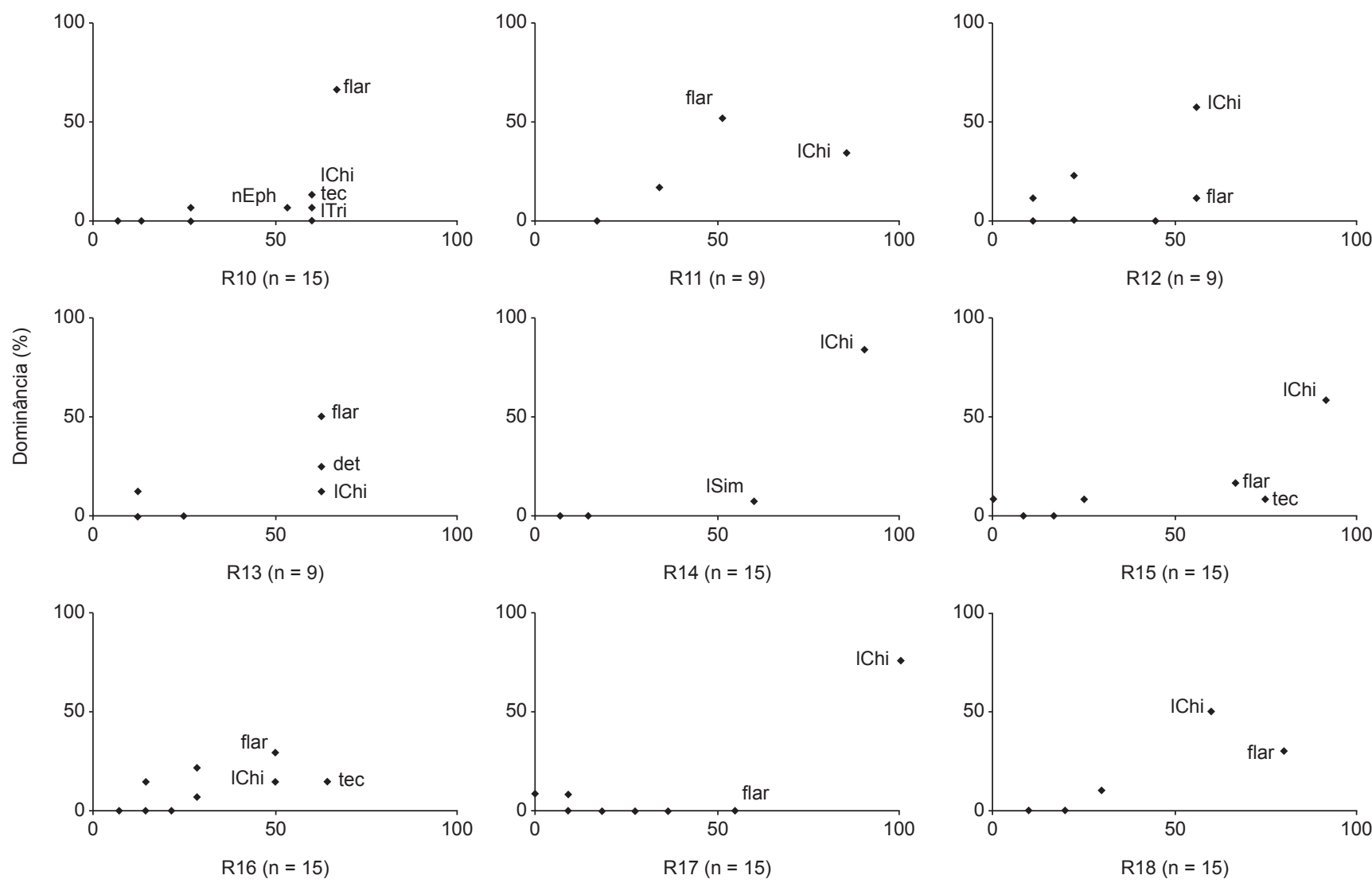

Frequência de ocorrência (\%)

Figura 3. Representação gráfica da dominância (eixo y) em função da freqüência de ocorrência (eixo x) dos itens alimentares registrados nos conteúdos gástricos de Aspidoras fuscogutattus nos riachos R10-R18 ( $\mathrm{n}=$ número de exemplares examinados): flar, fragmentos de larvas aquáticas de insetos; 1Chi, larvas aquáticas de Chironomidae; 1Sim, larvas aquáticas de Simuliidae; 1Tri, larvas de Trichoptera; nEph, ninfas de Ephemeroptera; tec, tecamebas; det, detritos.

Figure 3. Graphic representation of the dominance (axis $y$ ) in function to the frequency of occurrence (axis $x$ ) of the feeding items registered in the stomach contents of Aspidoras fuscogutattus in the streams R10 to R18 ( $\mathrm{n}=$ number of examined specimens): flar, aquatic insect larvae debris; fveg, vegetal debris; 1Chi, aquatic Chironomidae larvae; 1Cer, aquatic Ceratopogonidae larvae.

morfológicas e comportamentais devem ser explicar a habilidade de A. fuscoguttatus em explorar cursos d'água com fundos arenosos, tornando-a resistente às modificações do hábitat que gerem assoreamento, especialmente quando observamos a elevada abundância da espécie em riachos com elevados graus de deposição de sedimentos finos (Casatti et al. 2006, Ferreira \& Casatti 2006).

Chironomidae foi um item expressivo em praticamente todos os estômagos analisados e, portanto, pouco influenciado pelas diferentes condições ambientais. Contudo, de acordo com a ordenação gerada na NMDS, na dieta dos peixes de alguns riachos houve contribuição diferencial de detritos, tecamebas e fragmentos de vegetais superiores. Detrito foi um item com menos de $6 \%$ de representatividade na dieta dos peixes de modo geral e com contribuição máxima de $26 \%$ nos riachos em que ocorreu. De modo geral, a ocorrência deste item na dieta de $A$. fuscoguttatus provém de riachos estruturalmente muito simplificados, com leitos repletos de areia. É provável que a ingestão deste item tenha ocorrido acidentalmente enquanto os peixes se alimentavam de presas bentônicas, visto que ocorreu em baixas quantidades; nestas condições, detrito pode ser considerado um item nutricionalmente pouco importante (Bowen 1984). Outros estudos realizados na região também registraram hábitos detritívoros em algumas espécies bentônicas especialistas, como os cascudos Hypostomus ancistroides e H. nigromaculatus, geralmente perifitívoros em ambientes menos degradados (Ferreira \& Casatti 2006).

Tecamebas podem estar presentes não só em ambientes conservados, mas também onde há aporte de esgoto e assoreamento (Fulone et al. 2005), devendo, portanto ser um item comum em riachos, apesar de pouco conhecido. Como no presente estudo tecamebas foi um item associado à dieta de peixes em riachos de águas mais claras e com maior variedade de estruturas ripárias (árvores e arbustos), pode-se dizer que essas condições facilitariam sua captura por indivíduos de A. fuscoguttatus.

Fragmentos de vegetais superiores foram relativamente expressivos (8-26\% de ocorrência numérica) na dieta dos peixes de alguns riachos com extensos bancos de gramíneas junto às margens (R1, R6, R14), podendo sugerir que estes fragmentos sejam obtidos acidentalmente, durante a captura de outras presas em meio à vegetação marginal. Coincidentemente, esses riachos também apresentaram águas relativamente mais turvas, sendo que o riacho R6 apresentou 511 NTUs de turbidez. Apesar da conhecida diminuição da habilidade visual para detecção das presas em águas turvas (Sweka \& Hartman 2001, White \& Harvey 2007), é provável que a alimentação de 


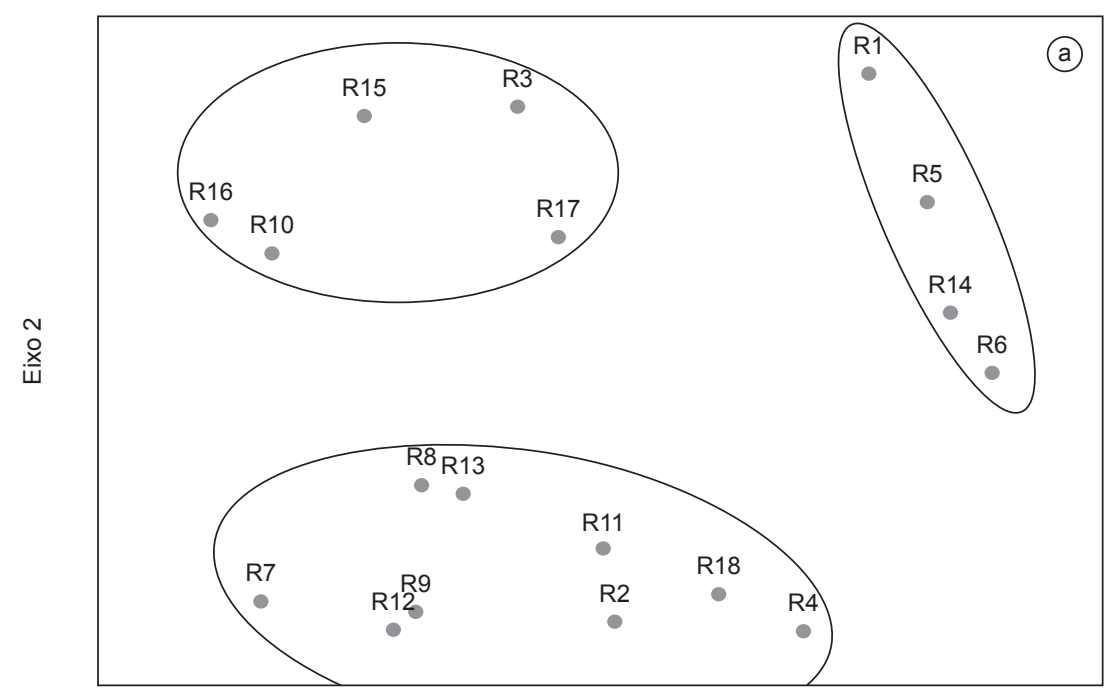

Eixo 1
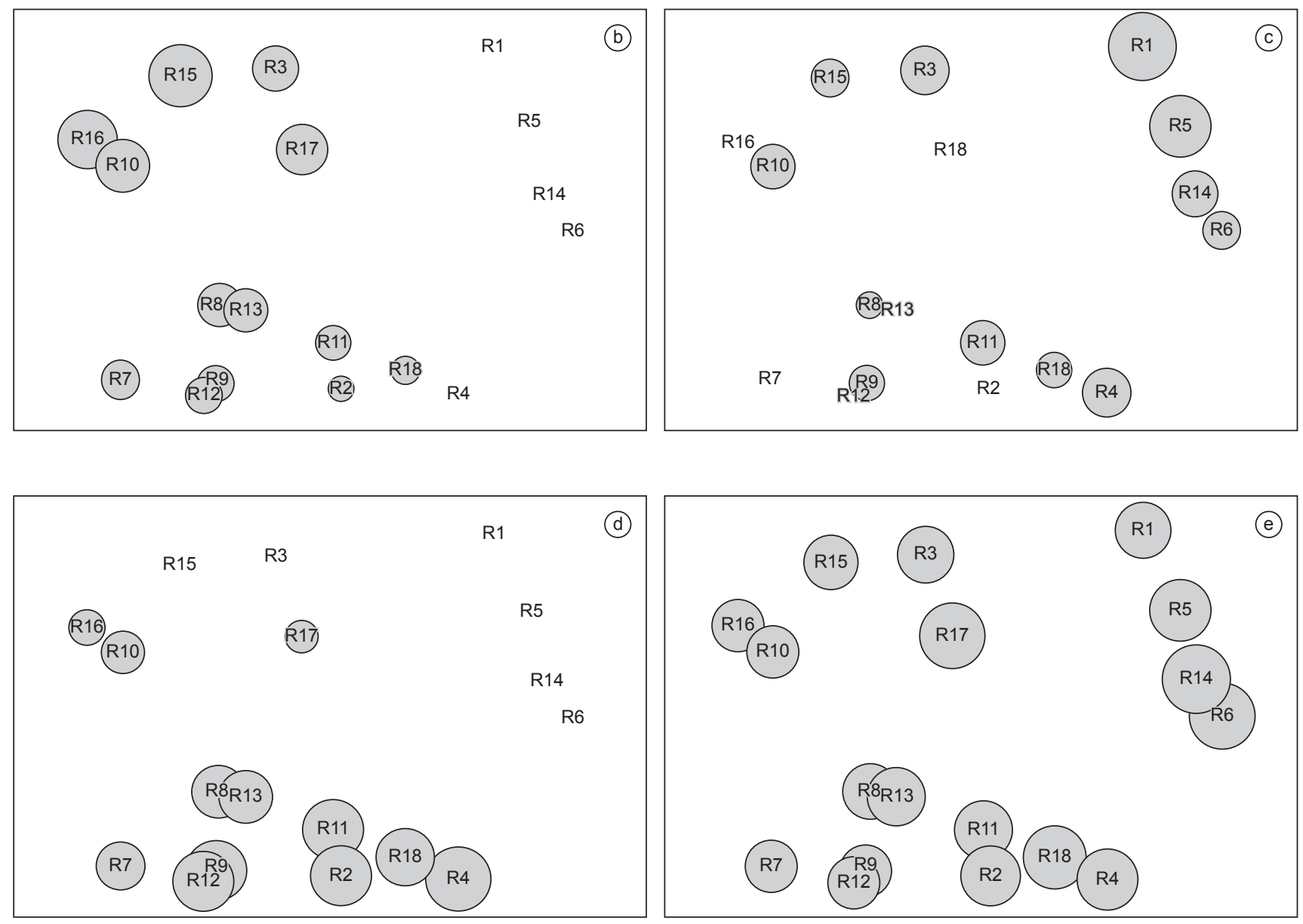

Figura 4. a) Representação gráfica da ordenação resultante da análise de escalonamento multidimensional não métrica (NMDS) da dieta de Aspidoras fuscoguttatus. b-e) Contribuição (expressa conforme o tamanho do círculo) de (b) tecamebas, (c) fragmentos de vegetais, (d) detritos, (e) insetos aquáticos. Stress $=0,15$.

Figure 4. a) Graphic representation of the non-metric multidimensional scaling analysis (NMDS) of the diet of Aspidoras fuscoguttatus. b-e) Contribution (expressed according to the circle size) of (b) testate amoebas, (c) vegetal debris, (d) detritus, (e) aquatic insects. Stress $=0.15$. 
Tabela 2. Resultados da análise de similaridade (ANOSIM) considerando a influência de 12 descritores abióticos sobre a dieta de Aspidoras fuscoguttatus em 18 riachos da região noroeste do estado de São Paulo, sistema do Alto Rio Paraná. Valores de R próximos a zero indicam aceite da hipótese nula; quanto menor o valor de $\mathrm{p}$ menor a probabilidade de ocorrência de erro Tipo I. Em negrito está apontado o descritor com maior influência sobre a dieta da espécie.

Table 2. Results of similarity analysis (ANOSIM) considering the influence of 12 abiotic descriptors on the Aspidoras fuscoguttatus diet in 18 streams, São Paulo State northwester region, upper Rio Paraná system. R values close to zero indicate null hypothesis acceptance; as low the $\mathrm{p}$ value low is tem probability of Type I error occurrence. In bold is indicated the descriptor with the highest influence on the diet species.

\begin{tabular}{lcc}
\hline \multicolumn{1}{c}{ Descritores } & $\mathbf{R}$ & $\mathbf{p}$ \\
\hline \multicolumn{1}{c}{ Substrato } & & \\
Argila (\%) & $-0,082$ & 76,1 \\
Areia (\%) & $-0,097$ & 77,4 \\
Cascalho (\%) & 0,16 & 10 \\
Rochas (\%) & 0,362 & 2 \\
Lage (\%) & 0,36 & 5,6 \\
$\quad$ Elementos do hábitat interno & & \\
Troncos e galhos (\%) & 0,071 & 50 \\
Macrófitas (\%) & $-0,035$ & 58,9 \\
$\quad$ Elementos ripários & & \\
Arbustos (\%) & $-0,03$ & 59,6 \\
Árvores (\%) & $-0,119$ & 77,8 \\
$\quad$ Outros & & \\
Correnteza (m/s) & 0,087 & 18,8 \\
Área (m ${ }^{2}$ ) & $-0,816$ & 90,8 \\
Turbidez (NTU) & 0,858 & 1,8 \\
Profundidade (m) & 0,153 & 34,1 \\
\hline
\end{tabular}

A. fuscogutattus seja pouco afetada em tais condições, pois os indivíduos localizam suas presas revolvendo o substrato com auxílio de barbilhões táteis e, portanto, com pouca dependência da visão. Dessa forma, enquanto que em águas mais claras os itens alimentares podem ser visualmente selecionados pelos peixes, em águas mais turvas essa seleção não ocorre, mas ainda assim, não deve haver prejuízo à alimentação dos indivíduos, em razão da presença de barbilhões sensoriais que os auxiliam na localização das presas.

Apesar da contribuição de outros itens alimentares, que varia conforme determinadas características do ambiente, larvas e ninfas aquáticas de insetos, notadamente de Chironomidae, parecem ser organismos abundantes mesmo em ambientes moderadamente impactados (Callisto et al. 2001), sendo um recurso altamente disponível para peixes invertívoros, como é o caso de A. fuscoguttatus. Este fator, juntamente com a habilidade em obter recursos em riachos com leitos arenosos e águas turvas, podem compor parte das explicações para a elevada abundância e ampla ocorrência da espécie na região.

\section{Agradecimentos}

Agradecemos à equipe do Laboratório de Ictiologia DZSJRP-IBILCE pelo auxílio no campo; a Sirlei T. Bennemann, Renato B. Araujo, Fabrício B. Teresa e revisores pela leitura crítica do manuscrito e ao IBAMA pela licença de coleta (001/2003). Este trabalho foi financiado pela Fundação de Amparo à Pesquisa do Estado de São Paulo (FAPESP) dentro do Programa BIOTASP/FAPESP (www.biota.org.br) - O Instituto Virtual da Biodiversidade (processos 01/13340-7, 05/00901-1). LC é bolsista de Produtividade CNPq, CPF é bolsista da FAPESP.

\section{Referências}

ARANHA, J.M.R., CARAMASCHI, E.P. \& CARAMASCHI, U. 1993. Ocupação espacial, alimentação e época reprodutiva de duas espécies de Corydoras Lacépède (Siluroidei, Callichthyidae) coexistentes no Rio Alambari (Botucatu, São Paulo). Revta. Bras. Zool. 10(3):453-466.

ARANHA, J.M.R., TAKEUTI, D.F. \& YOSHIMURA, T.M. 1998. Habitat use and food partitioning of the fishes in a coastal stream of Atlantic Forest, Brazil. Rev. Biol. Trop. 46(4):951-959.

ARAUJO, R.B. \& GARUTTI, V. 2002. Biologia reprodutiva de Aspidoras fuscoguttatus (Siluriformes, Callichthyidae) em riacho de cabeceira da bacia do alto rio Paraná. Iheringia Sér. Zool. 92(4):89-98.

ARAUJO, R.B. \& GARUTTI, V. 2003. Ecology of a stream from upper Paraná river basin inhabited by Aspidoras fuscoguttatus Nijssen \& Isbrücker, 1976 (Siluriformes, Callichthyidae). Braz. J. Biol. 63(3):363-372.

ARID, F.M. 1973. Comportamento espacial da formação Bauru na região Norte-Ocidental do Estado de São Paulo. Rev. Bras. Geoc. 3(1):23-24.

BARCHA, S.F. \& ARID, F.M. 1971. Estudo de evapotranspiração na região Norte-Ocidental do Estado de São Paulo. Rev. Ciênc. Fac. Ciênc. Let. 1:99-122.

BENNEMANN, S.T., CASATTI, L. \& OLIVEIRA, D.C. 2006. Alimentação de peixes: proposta para análise de itens registrados em conteúdos gástricos. Biota Neotrop. 6(2) http://www.biotaneotropica.org.br/v6n2/ pt/abstract?article+bn01206022006 (último acesso em 20/02/2007).

BOWEN, S.H. 1984. Detritivory in Neotropical fish communities. In Evolutionary ecology of Neotropical freshwater fishes (T.M. Zaret, ed.). Dr. W. Junk Publishers, The Hague, p. 59-66.

BRITTO, M.R. 1998. Two new species of the genus Aspidoras (Siluriformes: Callichthyidae) from central Brazil. Ichthyol. Explor. Freshwaters, 8(4):359-368.

BRITTO, M.R. 2000. Aspidoras depinnai (Siluriformes: Callichthyidae): a new species from northeastern Brazil. Copeia, 2000(4):1048-1055.

BRITTO, M.R., LIMA, F.C.T. \& MOREIRA C.R. 2002. Aspidoras velites, a new catfish from the upper Rio Araguaia basin, Brazil (Teleostei Siluriformes: Callichthyidae). Proc. Biol. Soc. Wash. 115(4):727-736.

BRITTO, M.R., LIMA, F.C.T. \& SANTOS, A.C. 2005. A new Aspidoras (Siluriformes: Callichthyidae) from rio Paraguaçu basin, Chapada Diamantina, Bahia, Brazil. Neotrop. Ichthyol. 3(4):473-479.

BURGESS, W.E. 1989. An atlas of freshwater and marines catfishes. T.F.H. Publications, Boca Ratón.

CALLISTO, M., MORETTI, M. \& GOULART, M. 2001. Macroinvertebrados bentônicos como ferramenta para avaliar a saúde de riachos. Rev. Bras. Rec. Hídr. 6(1):71-82.

CARVALHO, E.M. \& UIEDA, 2004. Colonização por macroinvertebrados bentônicos em substrato artificial e natural em um riacho da serra de Itatinga, São Paulo, Brasil. Revta. Bras. Zool. 21(2):287-293.

CARVALHO, E.M. \& UIEDA, V.S. 2006. Colonization routes of benthic macroinvertebrates in a stream in southeast Brazil. Acta Limnol. Bras. 18(4):367-376.

CASATTI, L. 2002. Alimentação dos peixes em um riacho do Parque Estadual Morro do Diabo, bacia do alto rio Paraná, sudeste do Brasil. Biota Neotrop. 2(2): http://www.biotaneotropica.org.br/v2n2/pt/ abstract?article+BN02502022002 (último acesso em 20/02/2007).

CASATTI, L., LANGEANI, F., SILVA, A.M. \& CASTRO, R.M. 2006. Stream fishes, water and habitat quality in a pasture dominated basin, southeastern Brazil. Braz. J. Biol. 66(2B):681-699.

CLARKE, K.R. \& GORLEY, R.N. 2006. Primer v6: user manual/tutorial. Plymouth Marine Laboratory, Plymouth.

CLARKE, K.R. \& WARWICK, R.M. 2001. Change in marine communities: an approach to statistical analysis and interpretation. Plymouth Marine Laboratory, Plymouth.

CLARO Jr., L., FERREIRA, E., ZUANON, J.A. \& ARAUJO-LIMA, C. 2004. O efeito da floresta alagada na alimentação de três espécies de peixes onívoros em lagos de várzea da Amazônia central, Brasil. Acta Amaz. 34(1):133-137. 
CURIO, E. 1976. The ethology of predation. Springer, Berlin.

FERREIRA, C.P. \& CASATTI, L. 2006. Influência da estrutura do hábitat sobre a ictiofauna de um riacho em uma micro-bacia de pastagem, São Paulo, Brasil. Revta. Bras. Zool. 23(3): 642-651.

FULONE, L.J., LIMA, A.F., ALVES, G.M., VELHO, L.F.M. \& LANSACTÔHA, F.A. 2005. Composição de amebas testáceas (ProtozoaRhizopoda) de dois córregos do Estado de São Paulo, incluindo novos registros para o Brasil. Acta Sci. Biol. Sci. 27(2):113-118.

GARUTTI, V. 1988. Distribuição longitudinal da ictiofauna em um córrego da região noroeste do Estado de São Paulo, Bacia do Rio Paraná. Rev. Bras. Biol. 48(4):747-759.

GELWICK, F.P. \& MATTHEWS, W.J. 1996. Trophic relations of stream fishes. In Methods in stream ecology (G. Lamberti \& R. Hauer, eds.). Academic Press, New York, p. 475-492.

HAHN, N.S., ALMEIDA, V.L.L. \& GASPAR Da LUZ, K.D. 1997. Alimentação e ciclo alimentar diário de Hoplosternum littorale (Hancock) (Siluriformes, Callichthyidae) nas lagoas Guaraná e Patos da planície do alto rio Paraná, Brasil. Revta Bras. Zool. 14(1):57-64.

HAY, C.H., FRANTI, T.G., MARX, D.B., PETERS, E.J. \& HESSE, L.W. 2008. Macroinvertebrate drift density in relation to abiotic factors in the Missouri River. Hydrobiol. 598(1):175-189.

HYNES, H.B.N. 1950. The food of fresh-water sticklebacks (Gasterosteus aculeatus and Pygosteus pungitius), with a review of methods used in studies of the food of fishes. J. Anim. Ecol. 19(1):36-57.

LOWE-McCONNELL, R.H. 1987. Ecological studies in tropical fish communities. Cambridge, Cambridge University Press.

MENEZES, N.A., CASTRO, R.M.C., WEITZMAN, S.H. \& WEITZMAN, M.J. 1990. Peixes de riacho da Floresta Atlântica Costeira Brasileira: um conjunto pouco conhecido e ameaçado de vertebrados. In II Simpósio de ecossistemas da Costa Sul e Sudeste Brasileira: estrutura, função e manejo. Academia de Ciências do Estado de São Paulo, 290-295.

NOVAES, J.R.P. 2007. Campeões de produtividade: dores e febres nos canaviais paulistas. Estud. av. 21(59):167-177.

NIMER, E. 1989. Climatologia do Brasil. Secretaria de Planejamento e Coordenação da Presidência da República; IBGE, Rio de Janeiro.

REIS, R.E., KULLANDER, S.O. \& FERRARIS Jr., C.J. 2003. Check list of the freshwater fishes of South and Central America. EDIPUCRS, Porto Alegre.

ROSENFELD, J. 2003. Assessing the habitat requirements of stream fishes: an overview and evaluation of different approaches. Trans. Am. Fish. Soc. 132(5):953-968.

SAZIMA, I. 1986. Similarities in feeding behaviour between some marine and freshwater fishes in two tropical communities. J. Fish Biol. 29(1):53-65.

Secretaria do Meio Ambiente; Instituto Florestal - SMA; IF. 2005. Inventário florestal do Estado de São Paulo. Imprensa Oficial do Estado de São Paulo, São Paulo.

SWEKA, J.A. \& HARTMAN, K.J. 2001. Effect of turbidity on prey consumption and growth in brook trout and implications for bioenergetics modeling. Can. J. Fish. Aquat. Sci. 58(2):386-393.

WHITE, J.S. \& HARVEY, B.C. 2007. Winter feeding success of stream trout under different streamflow and turbidity conditions. Trans. Am. Fish. Soc. 136:1187-1192.

Recebido em 19/05/08

Versão reformulada recebida em 10/11/08

Publicado em 18/02/09 
Alimentação de Aspidoras

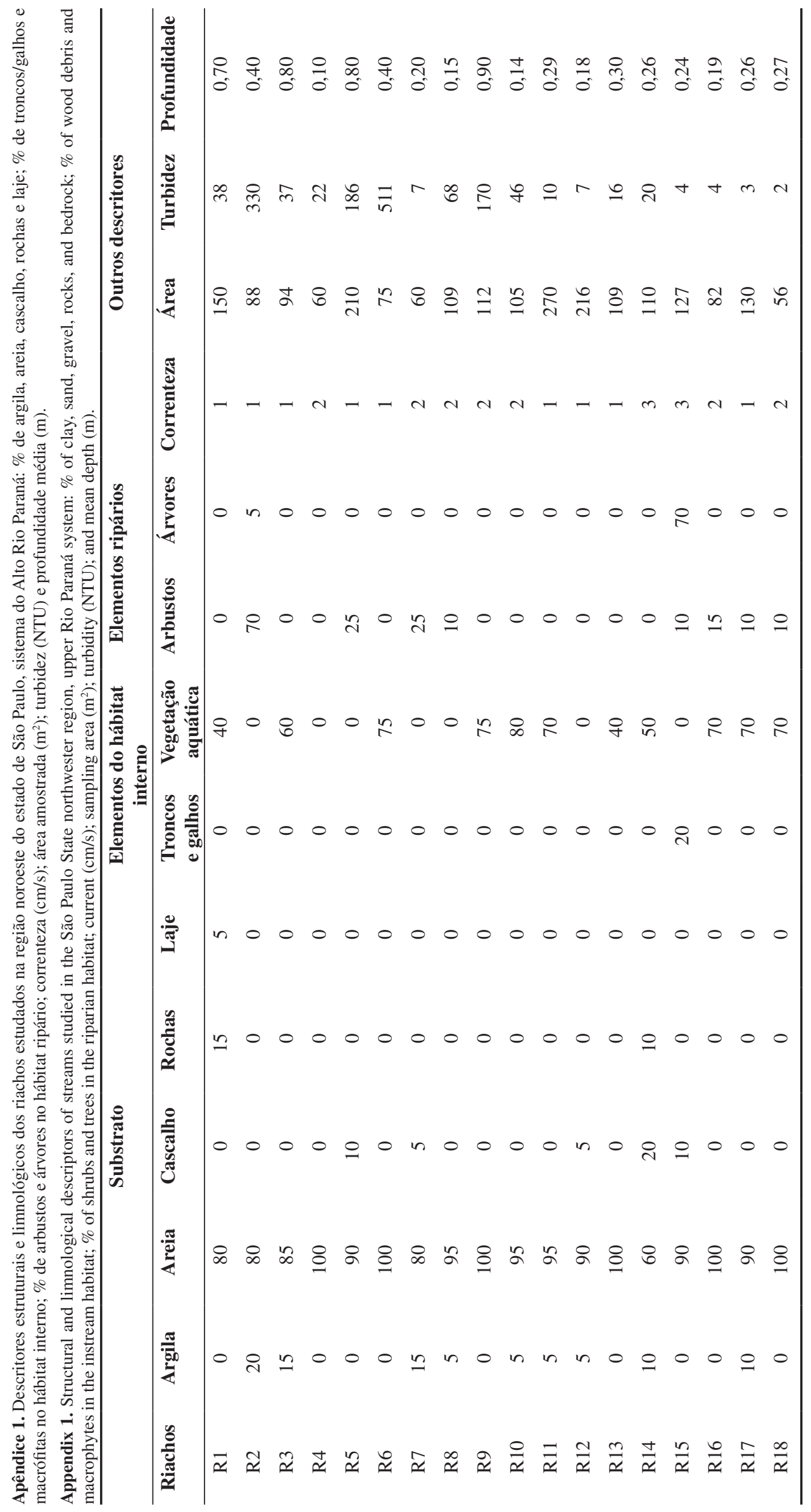


